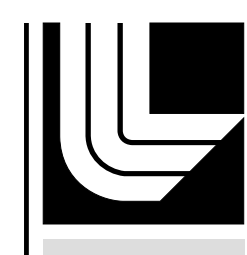

LAWRENCE LIVERMORE N A T IO N A L LABORATORY

\title{
Stochastic Transport Modeling of Resonant Magnetic Perturbations in DIII-D
}

I. Joseph, R. A. Moyer, T. E. Evans, M. J. Schaffer, A. M. Runov, R. Schneider, S. V. Kasilov, M. Groth, M. E. Fenstermacher

June 4, 2006

Journal of Nuclear Materials 
This document was prepared as an account of work sponsored by an agency of the United States Government. Neither the United States Government nor the University of California nor any of their employees, makes any warranty, express or implied, or assumes any legal liability or responsibility for the accuracy, completeness, or usefulness of any information, apparatus, product, or process disclosed, or represents that its use would not infringe privately owned rights. Reference herein to any specific commercial product, process, or service by trade name, trademark, manufacturer, or otherwise, does not necessarily constitute or imply its endorsement, recommendation, or favoring by the United States Government or the University of California. The views and opinions of authors expressed herein do not necessarily state or reflect those of the United States Government or the University of California, and shall not be used for advertising or product endorsement purposes. 


\title{
Stochastic transport modeling of
}

\section{resonant magnetic perturbations in DIII-D}

\author{
I. Joseph ${ }^{a *}$, R.A. Moyer ${ }^{\mathrm{a}}$, T.E. Evans ${ }^{\mathrm{b}}$, M.J. Schaffer ${ }^{\mathrm{b}}$, A.M. Runov ${ }^{\mathrm{c}}$, \\ R. Schneider ${ }^{c}$, S.V. Kasilov', M. Groth ${ }^{\mathrm{e}}$, and M.E. Fenstermachere \\ aUniversity of California-San Diego, La Jolla, California, USA \\ bGeneral Atomics, P.O. Box 85608, San Diego, California, USA \\ cMax Planck Institut für Plasmaphysik, EURATOM Association, Teilinstitut Greifswald, Germany \\ dinstitute of Plasma Physics, Kharkov Institute of Physics and Technology, Kharkov, Ukraine \\ e'Lawrence Livermore National Laboratory, Livermore, California, USA
}

Three-dimensional two-fluid simulations of heat transport due to resonant magnetic perturbations of tokamaks have been computed by coupling the TRIP3D field line tracing code to the E3D edge transport code. The predicted electron temperature contours follow the new separatrix represented by the perturbed invariant manifold structure of the X-point in qualitative agreement with X-point TV observations. However, preliminary modeling predicts that the resulting stochastic heat transport is greater than that measured in low-collisionality ELM suppression experiments in DIII-D H-mode plasmas. While improved determination of transport coefficients is definitely required, possible explanations include plasma screening of resonant perturbations, invalid treatment of the edge as a fluid, or insufficient understanding of stochastic heat transport.

JNM Keywords: M0200, P0500, P0600, T0100

PSI Keywords: DIII-D, 3D fluid code, edge modeling, stochastic boundary, ELM control PACS: 05.45.Gg, 52.55.Fa, 52.55.Rk, 52.65.-y 
*Corresponding author: Ilon Joseph, General Atomics, P.O. Box 85608, MS 13-368, San Diego,

California 92186-5608

e-mail: ijoseph@ucsd.edu

Presenting author and e-mail: Same as Corresponding author 


\section{Introduction}

Recent DIII-D experimental results [1-4] have shown that the addition of small resonant magnetic perturbations (RMPs), $\langle\delta b / B\rangle_{\text {res }} \sim 10^{-4}$, to the edge of a tokamak can suppress edge localized mode (ELM) instabilities in H-modes over a wide range of collisionality. In order to provide detailed modeling of the effect of the potentially stochastic fields on DIII-D equilibria, a new tool for calculating fully three-dimensional heat transport in resonantly perturbed tokamaks has been created by coupling the TRIP3D magnetic field line tracing code [5] to the E3D two-fluid transport code [6,7]. Although more complete physical modeling is still necessary, such calculations could be used to predict plasma profiles and, in turn, assess the performance of RMP-based ELM suppression strategies for new devices like ITER.

TRIP3D models the magnetic field as a super-position of the axisymmetric equilibrium fields calculated by the EFIT Grad-Shafranov solver [8] and the non-axisymmetric fields produced by models of DIII-D's measured field errors, error-correction coils (C-coil), and MHD control coils (I-coil). Under typical, $n=3$, even-parity, I-coil operating conditions characteristic of the low-collisionality ELM control experiments $[3,4]$, the relatively strong RMPs produce stochasticity over the outer $25 \%$ of the flux surfaces and allow field lines to directly escape over the last 5-10\% of flux surfaces (cf. Fig. 1). E3D computes the detailed electron and ion temperature profiles resulting from the 3D magnetic geometry specified by TRIP3D field model and an appropriate set of plasma/boundary conditions. E3D models the plasma as a quasi-neutral Braginskii two-fluid with anomalous perpendicular transport coefficients. The code has been used to model the TEXTOR tokamak's ergodic divertor [6] and to make predictions for the W7-X stellarator [7]. Aside from the specialized (MonteCarlo) numerical techniques required for accurate 3D computation, E3D works like a threedimensional version of the axisymmetric UEDGE [9] and B2 [10] edge-modeling codes. 


\section{Computational Results}

For our simulations, we focus on the electron temperature profiles in the low-density plasma discharge \#122342 at $4650 \mathrm{~ms}$ after ELMs have been completely eliminated by applying the $n=3$, even parity I-coil. Figure 1 shows a Poincaré plot of field line trajectories for 1000 toroidal transits produced by TRIP3D including the I-coil (3 kA) and C-coil (12 kAturns), but without intrinsic field-errors. Also shown are the EFIT flux surfaces, DIII-D vacuum vessel, and I-coil positions. The I-coil alone is found to have significant effect in the even-parity configuration; thus, we explore the changes to the temperature profile and heat flux deposition as the strength of the I-coil current is varied from 0 to $3 \mathrm{kA}$.

The TRIP3D magnetic field model superimposes the vacuum magnetic fields produced by the set of six C-coils and the six sets of upper and lower I-coils over an axisymmetric EFIT equilibrium. The field error module works in the same fashion, by superimposing the vacuum fields produced by the measured/inferred radial shifts and axial tilts of the poloidal and toroidal field coils and the toroidal field coil bus-work. The E3D code uses Monte-Carlo integration to solve the advection-diffusion equation for temperature by tracking the Brownian motion of heat packets. It efficiently treats the highly anisotropic heat conductivity that produces strong parallel transport by working in multiple local magnetic coordinate systems that follow the three-dimensional field line motion. Improved efficiency is obtained by using a set of local Boozer-like coordinates [7] which remove the majority of the parallel spatial dependence of the transport coefficients $\chi_{e, i}$. In the simulations, we use realistic wall geometry with sheath boundary conditions.

Since the density at the edge of an H-mode critically depends on the physics of neutral penetration [11], it is not modeled here. Instead, a fixed density profile is imposed which only roughly matches the experimental Thomson data where $n_{e}=2 \times 10^{19} \mathrm{~m}^{-3}$ on the inner surface, linearly drops to $n_{e}=4 \times 10^{18} \mathrm{~m}^{-3}$ near the EFIT separatrix, and is taken to be 
constant everywhere outside the separatrix. Since the actual density achieved varies with Icoil strength, we are not faithfully modeling actual experiments during the scan, but performing a hypothetical variation of the profiles.

As a fluid code, the region of validity is confined to the plasma edge; however, since the current E3D implementation requires the inner boundary to be a good closed flux surface, our inner boundary is set to the inner edge of the pedestal, a normalized poloidal flux of $\psi_{n}=$ 77\%, where we use fixed temperature boundary conditions: $T_{e}=1.6 \mathrm{keV}$ and $T_{i}=2.6 \mathrm{keV}$. Clearly, near this inner surface, the plasma is highly collisionless and the plasma may ultimately require a fully kinetic description. In the simulations below, we have chosen $\chi_{\perp, e}=\chi_{\perp, i}=0.2 \mathrm{~m}^{2} / \mathrm{s}$ constant throughout the domain. These values are found to match the experimental temperature profile from Thomson data reasonably well for the case of axisymmetric fields without perturbation and are consistent with previous experimental and computational studies of H-mode transport coefficients [12]. However, the coefficients are too small on the inner boundary resulting in a poor match to the measured input power. High fidelity modeling will require more reliable 1D and 2D transport analyses in order to better determine the experimentally inferred transport coefficients.

Under perturbation, the separatrix of a poloidally diverted tokamak becomes a so-called "homoclinic tangle" $[13,14]$. The simulations demonstrate that the temperature contours closely follow the invariant manifolds of the magnetic field (i.e., $\boldsymbol{B} \cdot \nabla \boldsymbol{T} \sim 0$ ) that form the tangle structure. Figure 2 compares the predicted electron temperature profile with the unstable manifold that exits the X-point calculated directly from field line mapping. Unlike density which is only advected along the direction of parallel flow, the temperature profile incorporates both branches of the tangle, and the stable manifold structure is clearly discernible in $T_{e}$ on the opposite side of the X-point. When the folds of this new separatrix grow large enough to intersect the divertor target plate at well-separated positions, they can 
cause the divertor strike point to split into multiple helical stripes. Experimental signatures of this phenomenon have been reported in Refs. 14-15. During $n=3$ even-parity I-coil current operation in shot \#123301, the $D_{\alpha}$ filtered tangential X-point TV image shown in Fig. 3 clearly displays multiple stripes on the inner strike point.

TRIP3D/E3D predictions for the electron temperature across the pedestal are shown as a function of normalized poloidal flux, $\psi_{n}$, in Figs. 4 and 5 as the I-coil current is successively raised from 0 to $3 \mathrm{kA}$. The electron temperature profile clearly cools and relaxes over the stochastic region as the perturbation strength is raised and shows that large temperature gradients can only be sustained over regions with good flux surfaces. In contrast, the measured $T_{e}$ profile, shown for the high coil current case, has a slightly higher pedestal and steeper gradient than the no-current case. The experimental input power was essentially unchanged after energizing the I-coil, while the simulated input power was increased by a factor of 2.5 as the I-coil was turned on to full strength. The average heat flux through the divertor legs increases by a factor of 2 while the width of the divertor footprints increase by a factor of 1.25; thus, the predicted thermal conductance of the edge + divertor plasma increased by a factor of 2.5. The lowest curve in Fig. 5 (purple) shows the effect of adding the C-coil at a current of $12 \mathrm{kA}$-turns (about $1 \mathrm{~m}$ from the plasma). Since the flux surfaces are destroyed outside of $\psi_{n}=65 \%$ as shown in the Poincaré plot in Fig. 1, the boundary conditions for this run correspond to fixed temperatures at $\psi_{n}=63 \%$.

\section{Discussion}

In contrast to the original high-collisionality experiments $[1,2]$ where large Type-I ELMs were replaced by a small transport event regime (Type-II ELMs?), the more recent lowcollisionality experiments [3,4] achieved complete ELM suppression. Since roughly the same stored energy was achieved for the same neutral beam injection (NBI) power, the energy losses due to ELMs must be replaced by another type of transport process either directly or 
indirectly generated by the RMP. Complete ELM elimination is only observed within a

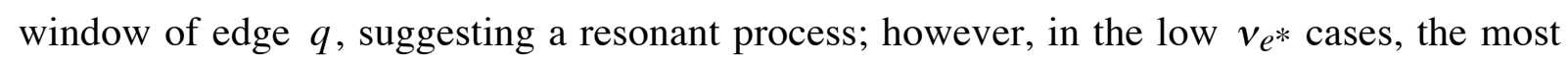
significant change to the measured Thomson profiles is a large drop in core density. In fact, in Ref. 3 it is shown that it is largely the drop in pedestal density that lowers the pressure gradient below the ELM stability threshold. The experimental electron temperature achieves the same pedestal height and its gradient actually increases across the transport barrier; however, accurate accounting for edge power balance may not necessarily indicate a drop in thermal conductivity.

The simulation results demonstrate that the temperature profiles closely follow the invariant manifolds [13] of the perturbed magnetic field and produce significant stochastic heat transport [16]. The calculated heat loss due to stochastic field line loss is too great to match the low-collisionality experimental results in Refs. 3-4. However, the current 3D simulations are still at the preliminary stages of adding the physics required to quantitatively model H-mode plasmas: parallel flow, electric field, neutral penetration, impurities, etc, and require more accurate determination of transport coefficients and specification of density profile. Ultimately, any model of RMP-induced transport must capture the clearest change in the experiment: increased particle transport. This may ultimately require the inclusion of neoclassical and/or nonlocal kinetic effects.

The simple approximation of adding the RMP fields in vacuum to an axisymmetric MHD equilibrium neglects the inductive response of the plasma to the applied fields. Shielding of resonant perturbations can occur due to plasma rotation [17], or more generally, by the parallel currents induced by nonideal force-balance [18]. On the other hand, if the plasma is close to a stability boundary, the applied fields can actually be amplified by the plasma response [19]. Rotation may be playing a crucial role, since these plasmas rotate quickly due to NBI heating, $\Omega_{\phi} \sim 5 \mathrm{kHz}$, and actually spin up as the density drops and the ELMs 
disappear. This contrasts with the high-collisionality experiments $[1,2]$ where rotation decreases after RMP application as expected. Future development will include plasma response models. In principle, self-consistency can be improved by iterating E3D with a code that is capable of determining the linear plasma response to a given set of perturbations.

The new E3D two-fluid code has been applied to 3D simulation of low-collisionality RMP ELM suppression experiments on DIII-D. In this work, we have focused solely on the geometric effect of chaotic magnetic field lines on thermal transport in the fluid regime. Improved fidelity to experimental conditions will require treatment of parallel flows, electric field, neutral penetration, impurities, and plasma rotation. The most important input, the radial magnetic field structure, will eventually need to be determined self-consistently. Quantitative treatment may eventually require kinetic modeling to accurately represent the nonlocal parallel heat transport in the collisionless pedestal. At the edge of the H-mode plasma, the complete response will be determined by the subtle interplay among rotation, turbulence, and ambipolar transport.

\section{Acknowledgments}

This work was supported by U.S. Department of Energy under DE-FC02-04ER54698, DE-FG02-05ER54809, DE-FG02-04ER54758 and W-7405-ENG-48. I. Joseph would also like to thank the Max Planck Insitut für Plasmaphysik and EURATOM for providing accommodations in Greifswald, Germany and for providing continued supercomputing support. This work was performed under the auspices of the U.S. Department of Energy by University of California, Lawrence Livermore National Laboratory under Contract W-7405-Eng-48. 


\section{References}

[1] R.A. Moyer, T.E. Evans, T.H. Osborne, et al., Phys. Plasmas 12 (2005) 056119.

[2] T.E. Evans, R.A. Moyer, J.G. Watkins, et al., Nucl. Fusion 45 (2005) 595.

[3] K.H. Burrell, T.E. Evans, E.J. Doyle, et al., Plasma Phys. Control. Fusion 47 (2005) B37.

[4] T.E. Evans, K.H. Burrell, R.A. Moyer, et al., Nature Phys. in press.

[5] T.E. Evans, R.A. Moyer, and P. Monat, Phys. Plasmas 9 (2002) 4957.

[6] A.M. Runov, D. Reiter, S.V. Kasilov, et al., Phys. Plasmas 8 (2001) 916.

[7] A.M. Runov, S. Kasilov, R. Schneider, et al., Contrib. Plasma Phys. 44 (2003) 18.

[8] L.L. Lao, H.E. St. John, R.D. Stambaugh, et al., Nucl. Fusion 25 (1985) 1611.

[9] T. Rognlien, J. Milovich, M. Rensink, and G. Porter, J. Nucl. Mater. 196-198 (1992) 347.

[10] B.J. Braams, P.J. Harbour, M.F.A. Harrison, et al. J. Nucl. Mater. 121 (1984) 75.

[11] R.J. Groebner, M.A. Mahdavi, A.W. Leonard, et al., Nucl. Fusion 44 (2004) 204.

[12] R.A. Moyer, J.W. Cuthbertson, T.E. Evans, et al., J. Nucl. Mater. 241-243 (1997) 633.

[13] V.K. Melnikov, Trans. Moscow Math. Soc. 12 (1963) 1.

[14] T.E. Evans, R.K.W. Roeder, J.A. Carter, et al., J. Phys. Conf. Ser. 7 (2005), 174.

[15] T.E. Evans, I. Joseph, R.A. Moyer, M.E. Fenstermacher, C.J. Lasnier, L. Yan, "Experimental and numerical studies of separatrix splitting and magnetic footprints in DIII-D," to be presented at the 17th Plasma Surface Interactions in Controlled Fusion Devices, Hefei, China, 2006.

[16] A.B. Rechester and M.N. Rosenbluth, Phys. Rev. Lett. 40 (1978) 38.

[17] R. Fitzpatrick, Phys. Plasmas 5 (1998) 3325.

[18] A.H. Boozer, Phys. Plasmas 12 (2005) 092504.

[19] A.H. Boozer, Phys. Rev. Lett. 86 (2001) 5059. 


\section{Figure captions}

Fig. 1. Poincaré plot of 10 TRIP3D magnetic field line trajectories for 1000 toroidal transits for shot \#122342 at 4650 ms with I-coil and C-coil, but no intrinsic field-errors. Also shown are the EFIT flux surfaces, EFIT separatrix, DIII-D inner vacuum vessel, and I-coil positions (red circles).

Fig. 2. The electron temperature contours computed by E3D closely follow the invariant manifolds that emanate from the X-point. The unstable manifold shown in black was computed directly from field line mapping. The stable manifold on the LFS is not shown.

Fig. 3. $D_{\alpha}$ filtered tangential X-point TV images show multiple stripes on the inner strike point during the $n=3$ I-coil current pulse in shot \#123301 at $2171 \mathrm{~ms}$.

Fig. 4. 2D electron temperature distribution predicted by E3D on the $\phi=0$ plane for varying levels of even-parity I-coil current: $0,1,2,3 \mathrm{kA}$. The separatrix tangle structure grows while the pedestal cools with increasing RMP strength.

Fig. 5. The electron temperature pedestal sampled at the experimental Thomson measurement location is predicted to cool as the I-coil current is scanned from 0 to $3 \mathrm{kA}$, as in Fig. 4 . The purple curve corresponds to the experimental settings: I-coil $=3 \mathrm{kA}$ and C-coil $=12 \mathrm{kA}$-turns shown in Fig. 1. 


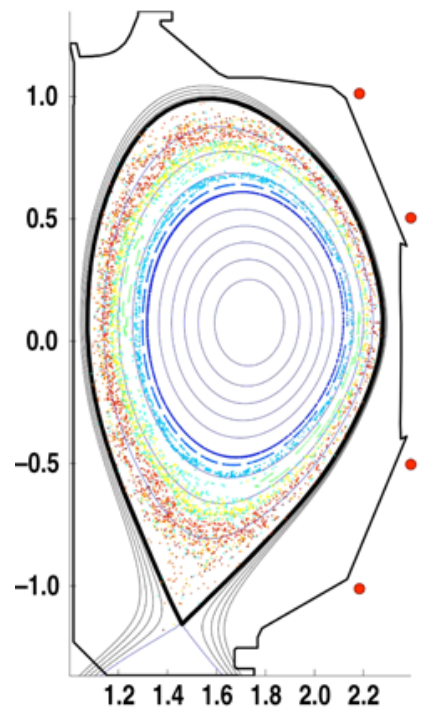

Fig. 1, I. Joseph et al., P3-11 


$$
0
$$




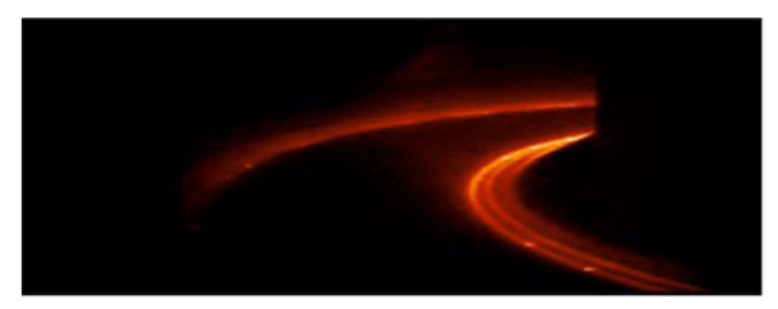

Fig. 3, I. Joseph et al., P3-11 


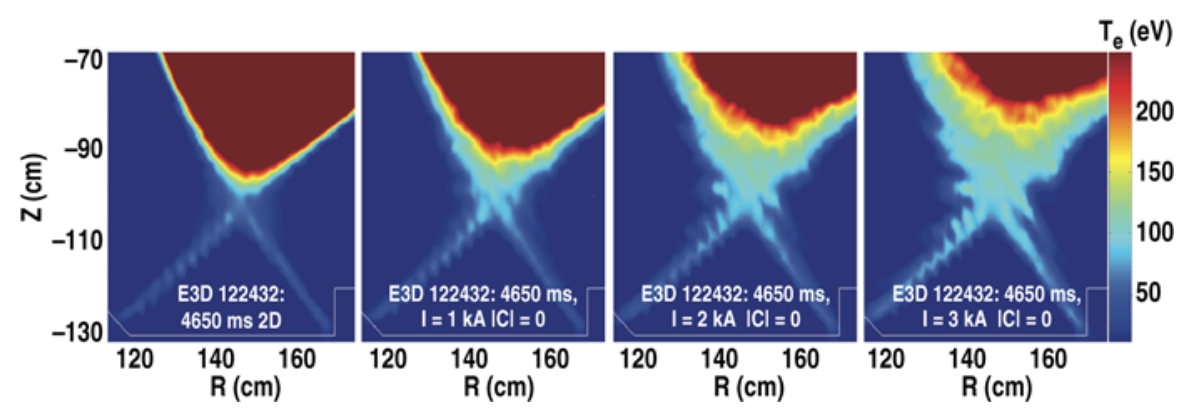

Fig. 4, I. Joseph et al., P3-11 


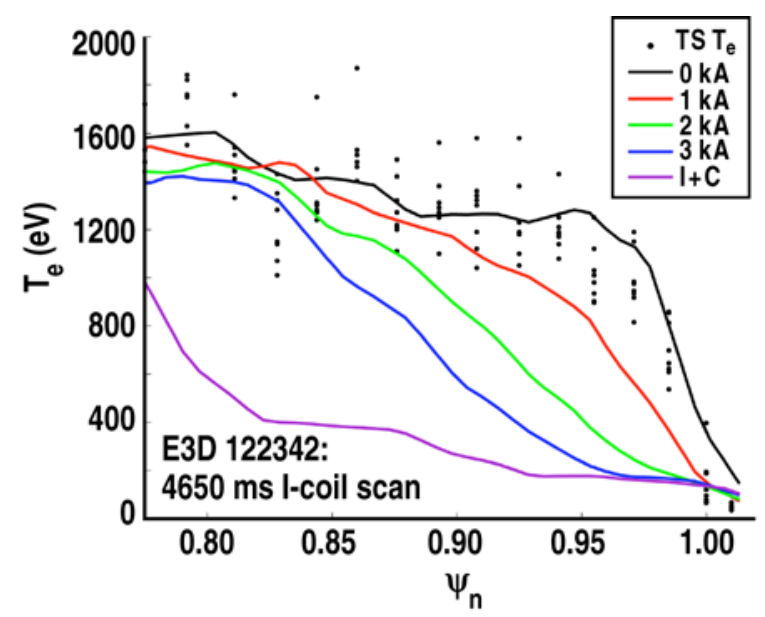

Fig. 5, I. Joseph et al., P3-11 